\title{
Risk of esophageal adenocarcinoma in patients with Barrett's esophagus using proton pump inhibitors: a systematic review with meta-analysis and sequential trial analysis
}

\author{
Lunan $\mathrm{Li}^{1,2}$, Zhongsheng Cao ${ }^{1}$, Chenjing Zhang ${ }^{2}$, Wensheng Pan ${ }^{2}$ \\ ${ }^{1}$ BengBu Medical College, Bengbu, China; ${ }^{2}$ Department of Gastroenterology, Zhejiang Provincial People's Hospital, People’s Hospital of Hangzhou \\ Medical College, Hangzhou, China \\ Contributions: (I) Conception and design: L Li, Z Cao; (II) Administrative support: None; (III) Provision of study materials or patients: Zhejiang \\ Provincial people's Hospital; (IV) Collection and assembly of data: L Li, Z Cao, C Zhang; (V) Data analysis and interpretation: All authors; (VI) \\ Manuscript writing: All authors; (VII) Final approval of manuscript: All authors. \\ Correspondence to: Wensheng Pan. Department of Gastroenterology, Zhejiang Provincial People's Hospital, People's Hospital of Hangzhou Medical \\ College; Institute of Gastrointestinal Diseases, Hangzhou Medical college; Zhejiang Provincial Engineering Laboratory of Diagnosis, Treatment and \\ Pharmaceutical Development of Gastrointestinal Tract Tumors, Hangzhou 310014, China. Email: wspan223@163.com.
}

\begin{abstract}
Background: Proton pump inhibitors (PPIs) have been used to treat Barrett's esophagus (BE), but there seems to be insufficient evidence that PPIs can prevent esophageal adenocarcinoma (EAC) and high grade dysplasia (HGD). This study aimed to evaluate the effects of PPIs in BE patients.

Methods: PubMed and EMBASE were systematically searched. Stata13 and trial sequential analysis (TSA) software were used to carry out related statistics. Pooled odds ratio (OR) with 95\% confidence intervals (CIs) were calculated.

Results: Using PPIs to reduce the incidence of EAC and HGD has not been confirmed (OR, 0.61; 95\% CI, 0.29-1.26). The pooled results of three cohort studies reported that PPIs use was protective (OR 0.48; 95\% CI, 0.33-0.70). But the pooled results of five case-control study indicating PPIs use does not prove this protective effect (OR 0.73; 95\% CI, 0.21-2.48). On pooled analysis of 4 US studies 2 Netherlands, protective effect on development of EAC and HGD was noted (OR, 0.59; 95\% CI, 0.43-0.80) and (OR, 0.16; 95\% CI, $0.03-0.75)$.

Conclusions: According to the Meta analysis and TSA of existing studies, the protective effect of PPIs on the progression of BE patients to EAC and/or HGD has not been confirmed. TSA shows that more patients are needed before a clear conclusion can be reached.
\end{abstract}

Keywords: Proton pump inhibitors (PPIs); esophageal adenocarcinoma (EAC); high grade dysplasia (HGD); Barrett's esophagus (BE); meta-analysis; trial sequential analysis (TSA)

Submitted Dec 01, 2020. Accepted for publication Feb 26, 2021.

doi: $10.21037 /$ tcr-20-3362

View this article at: http://dx.doi.org/10.21037/tcr-20-3362

\section{Introduction}

The incidence of esophageal adenocarcinoma (EAC) has increased (1), with gastroesophageal reflux thought to be a major contributor to the occurrence of EAC and high grade dysplasia (HGD). Barrett's esophagus (BE) is one of the steps in which adverse events occur and is characterized by the squamous epithelium of the lower part of the esophagus being covered by the columnar epithelium. Gastric acid can be reduced by proton pump inhibitors (PPIs), which then can slow the BE process. At present, PPIs are recommended for the treatment of BE, but the evidence concerning PPIs' use to prevent the progression from BE to HGD and EAC seems to be insufficient. Several studies have reported that using PPIs may decrease the risk of HGD and EAC $(2,3)$. 
In contrast, other studies have suggested that PPI use can increase the risk of HGD and EAC $(2,4)$. The effects of PPIs on the risk of EAC and HGD in patients with BE thus remains controversial. Consequently, we conducted a meta-analysis and trial sequential analysis (TSA) to clarify PPI administration for EAC and HGD. We present the following article in accordance with the Preferred Reporting Items for Systematic Review and Meta-analysis (PRISMA) reporting checklist (5) (available at http://dx.doi. org/10.21037/tcr-20-3362).

\section{Methods}

\section{Data sources, search strategy, and study selection}

PubMed and EMBASE were searched through September 1, 2020, with some studies being manually searched to locate further resources. The comprehensive list of search terms is displayed in Appendix 1. All studies were selected by two independent reviewers (Lunan Li and Zhongsheng Cao). The articles reporting relative risk (RR) or odds ratio (OR), or that provided data for their calculations, were deemed eligible for inclusion. Conference abstracts were excluded. Inclusion in the meta-analysis was not otherwise restricted by study size or language. In cases where reviewers disagreed about the inclusion of an article, a consensus was achieved through group discussion.

\section{Data extraction and quality assessment}

Data from each included study were extracted by two independent reviewers (Lunan $\mathrm{Li}$ and Zhongsheng Cao). The required information included the first author's name, publication year, country, age distribution, sex distribution, and research type. Any disagreements concerning the data to be extracted were resolved through discussion to maintain consistent results. Each article's quality was independently assessed by two reviewers (Lunan Li and Zhongsheng Cao) using the Newcastle-Ottawa scale (NOS) $(6,7)$. Any discrepancies in the quality assessment were resolved via group discussion.

\section{Data analysis}

Stata 13 (StataCorp, College Station, TX, USA) was used for statistical analyses. TSA program version 0.9 beta was used to control random errors and evaluate inaccuracies. The heterogeneity was calculated with $\mathrm{I}^{2}$ statistic and valued greater than $75 \%$, indicating considerable heterogeneity (8). When heterogeneity was present, the random-effects model, as DerSimonian and Laird described, was used to calculate ORs and 95\% confidence intervals (CIs) (9). Subgroup analyses were conducted to evaluate results across different study types and countries. TSA was used to estimate the amount of information needed for the meta-analysis of conclusions and to evaluate whether or not the results had type I errors due to an insufficient number of studies included. There is a risk of random errors in a conventional meta-analysis that can arise from sparse data and repeated tests (10). The TSA depends on the quantification of the amount of information required. The random-effects model was used for our study. We calculated the OR with 95\% CI for each included trial. Using TSA was to keep the overall risk of type I errors at $5 \%$ and the power at $80 \%$. To calculate the required information size, we used $11 \%$ of the event incidence in the control group for meta-analysis.

\section{Results}

The study selection process is shown in Figure 1. A total of 8 studies ( 3 cohort studies and 5 case-control studies) comprising 7,053 patients were included (2,11-17). The characteristics of each study are presented in Table 1. The quality of the methodology included in the study ranged from medium to high (Table 2).

The meta-analysis of the 8 studies of BE patients did not completely confirm the use of PPIs to reduce the incidence of EAC and HGD (OR, 0.61; 95\% CI, 0.29-1.26) and showed significant heterogeneity $\left(\mathrm{I}^{2}=89.7 \%\right.$; Figure 2$)$. Studies examining the association between PPIs and the progression of EAC or HGD in BE patients were inconsistent in study design and study location. In 3 cohort studies that reported the risk of $\mathrm{BE}$ patient progression to HGD and EAC, PPI use was shown to be protective (OR, $0.48 ; 95 \%$ CI, 0.33-0.70; $\mathrm{I}^{2}=0.0 \%$; Figure 3). However, the 5 case-control studies, which comprised $5144 \mathrm{BE}$ patients, did not confirm that PPI use has a protective effect (OR, 0.73; 95\% CI, 0.21-2.48), with considerable heterogeneity being present across studies $\left(\mathrm{I}^{2}=94.0 \%\right)$ (Figure 3). The pooled analysis of 4 studies in a US subgroup suggested a protective effect against the development of EAC and HGD (OR, 0.59; 95\% CI, 0.43-0.80; I'=3.2\%; Figure 4). Another subgroup consisted of 2 studies from the Netherlands that assessed PPI's effect on the development of EAC and HGD in BE (OR, 0.16; 95\% CI, 0.03-0.75; $\mathrm{I}^{2}=90.2 \%$; Figure 4).

TSA (Figure 5) showed that the trial monitoring 


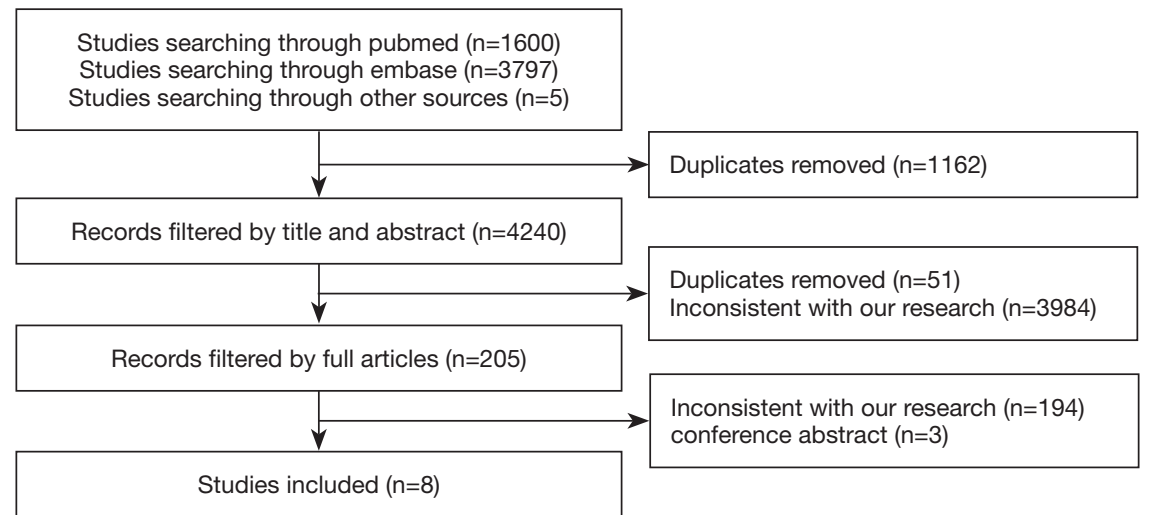

Figure 1 Flow diagram.

Table 1 Characteristics of studies included in meta-analysis and trial sequential analysis

\begin{tabular}{|c|c|c|c|c|c|c|c|}
\hline Author & Year & Country & \multicolumn{2}{|c|}{ Patients on PPI $(n=5,762)$} & \multicolumn{2}{|c|}{ Patients not on PPI $(n=1,291)$} & Research type \\
\hline de Jonge PJ & 2006 & Netherlands & 43 & 270 & 44 & 61 & Case-control study \\
\hline Nguyen DM & 2009 & America & 17 & 231 & 16 & 113 & Cohort study \\
\hline Nguyen DM & 2010 & America & 110 & 763 & 6 & 49 & Case-control study \\
\hline Hvid-Jensen F & 2014 & Denmark & 134 & 1,306 & 6 & 131 & Case-control study \\
\hline Masclee GM & 2015 & UK and Netherlands & 46 & 1,005 & 11 & 461 & Case-control study \\
\hline Thota PN & 2017 & America & 32 & 701 & 25 & 324 & Cohort study \\
\hline Tan MC & 2018 & America & 270 & 1,024 & 30 & 74 & Case-control study \\
\hline
\end{tabular}

PPIs, Proton pump inhibitors; EAC, Esophageal adenocarcinoma; HGD, High grade dysplasia.

Table 2 Quality assessment of studies included in meta-analysis and trial sequential analysis

\begin{tabular}{|c|c|c|c|c|c|}
\hline Study & Year & \multicolumn{4}{|c|}{ Study quality (NOS) } \\
\hline Nguyen DM & 2009 & 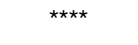 & $\star \star$ & 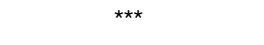 & 9 \\
\hline Nguyen DM & 2010 & $* \star \star *$ & ** & ** & 8 \\
\hline Hvid-Jensen F & 2014 & 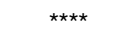 & ** & $* \star \star$ & 9 \\
\hline Masclee GM & 2015 & 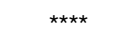 & ** & *** & 9 \\
\hline Thota PN & 2017 & $\star \star \star ~$ & ** & *** & 8 \\
\hline
\end{tabular}




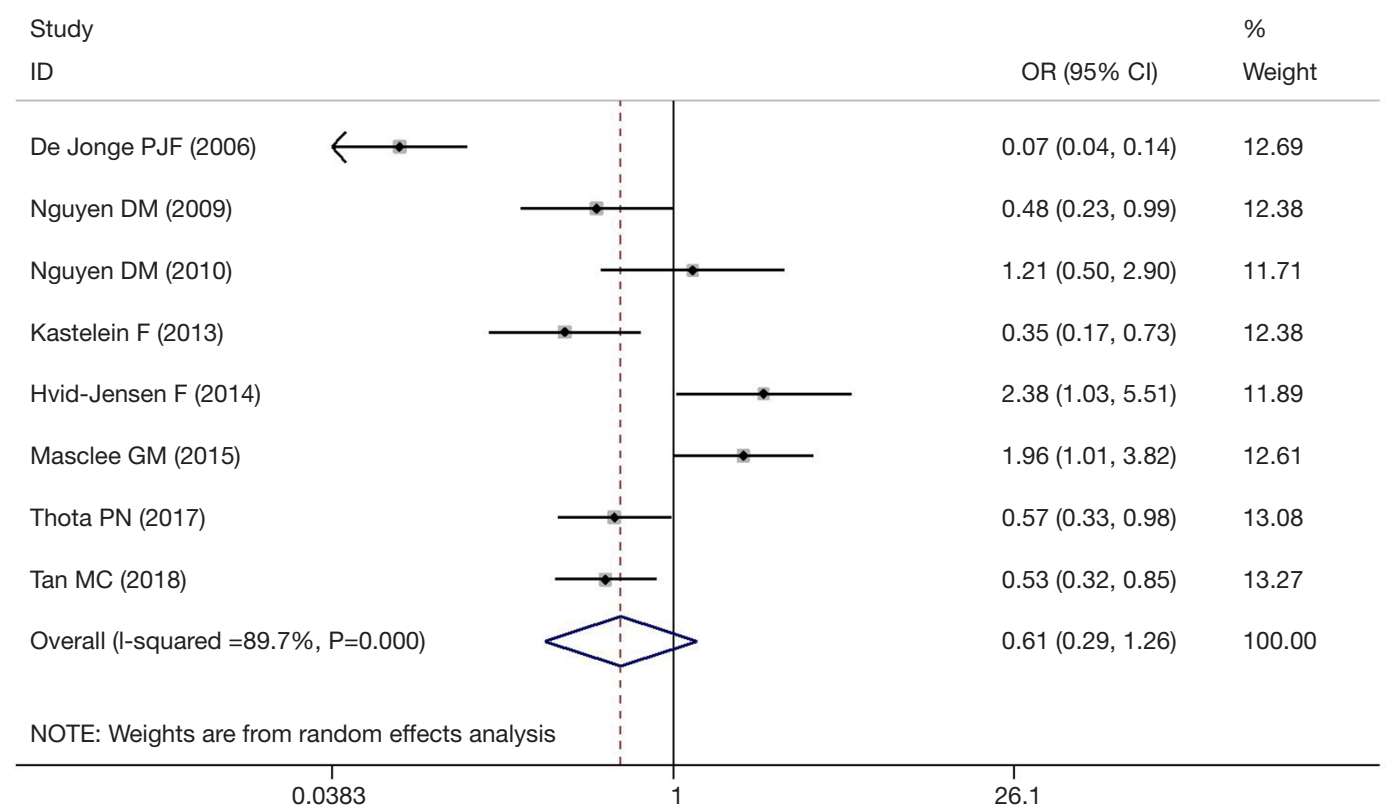

Figure 2 Pooled of the risk of EAC and/or HGD in patients with BE with PPIs exposure in included studies. EAC, esophageal adenocarcinoma; HGD, high grade dysplasia; BE, Barrett's esophagus; PPIs, Proton pump inhibitors.

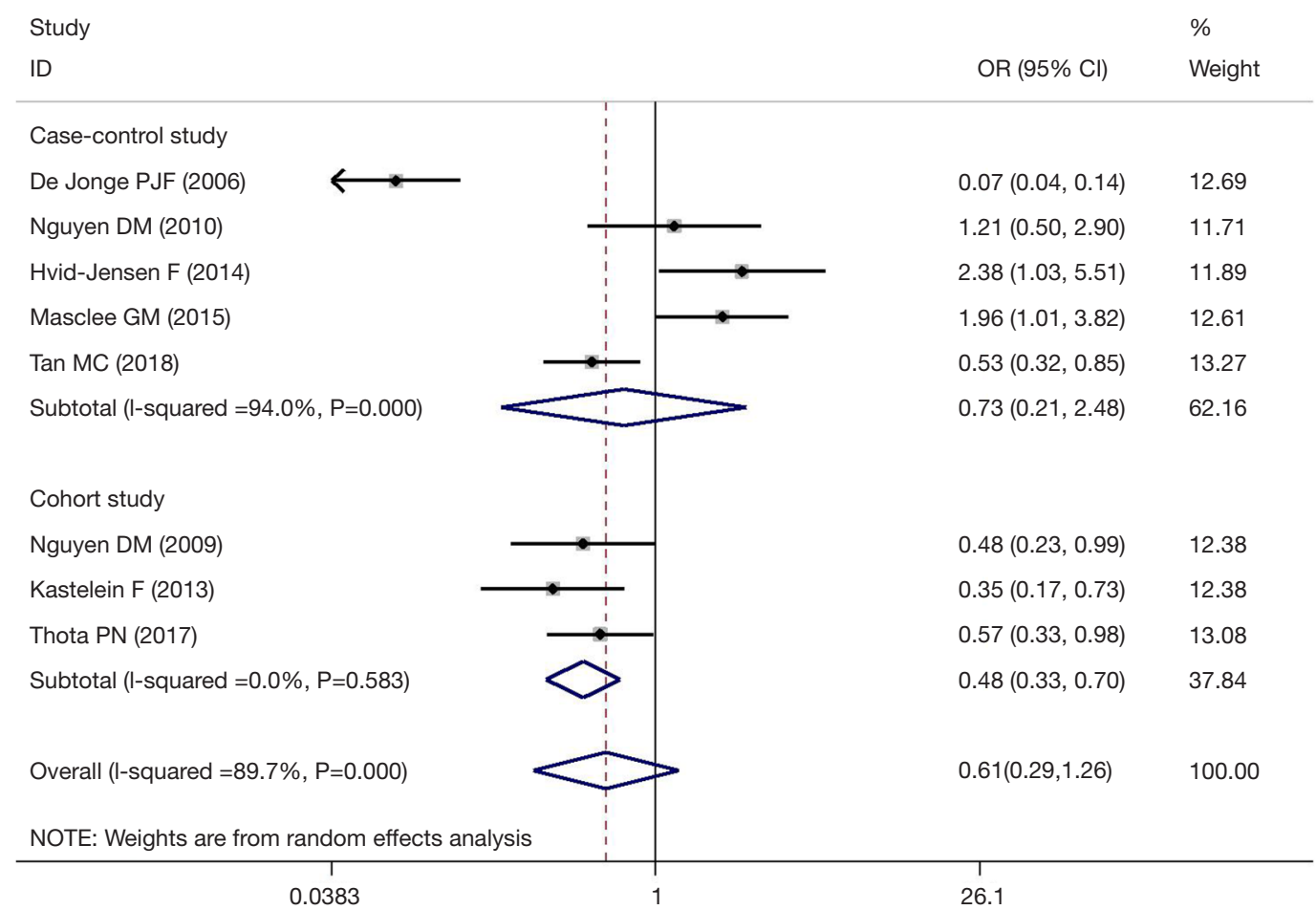

Figure 3 Subgroup analysis of the risk of patients EAC and/or HGD in patients with BE. EAC, esophageal adenocarcinoma; HGD, high grade dysplasia; BE, Barrett's esophagus. 


\begin{tabular}{|c|c|c|}
\hline Study & & $\%$ \\
\hline ID & OR $(95 \% \mathrm{Cl})$ & Weight \\
\hline Netherlands & & \\
\hline De Jonge PJF (2006) & $0.07(0.04,0.14)$ & 12.69 \\
\hline Kastelein F (2013) & $0.35(0.17,0.73)$ & 12.38 \\
\hline Subtotal $(\mathrm{I}$-squared $=90.2 \%, P=0$. & $0.16(0.03,0.75)$ & 25.06 \\
\hline America & & \\
\hline Nguyen DM (2009) & $0.48(0.23,0.99)$ & 12.38 \\
\hline Nguyen DM (2010) & $1.21(0.50,2.90)$ & 11.71 \\
\hline Thota PN (2017) & $0.57(0.33,0.98)$ & 13.08 \\
\hline Tan MG (2018) & $0.53(0.32,0.85)$ & 13.27 \\
\hline Subtotal $(\mathrm{I}$-squared $=3.2 \%, \mathrm{P}=0.376)$ & $0.59(0.43,0.80)$ & 50.44 \\
\hline Denmark & & \\
\hline Hvid-Jensen F (2014) & $2.38(1.03 .5 .51)$ & 11.89 \\
\hline subtotal (I-squared = .\%, P= .) & $2.38(1.03,5.51)$ & 11.89 \\
\hline UK and Netherlands & & \\
\hline Masclee GM (2015) & $1.96(1.01,3.82)$ & 12.61 \\
\hline Subtotal $(\mathrm{l}$-squared $=. \%, \mathrm{P}=)$. & $1.96(1.01,3.82)$ & 12.61 \\
\hline Overall (I-squared $=89.7 \%, P=0.000)$ & $0.61(0.29,1.26)$ & 100.00 \\
\hline NOTE: Weights are from random effects analysis & & \\
\hline $\begin{array}{c}1 \\
0.0339\end{array}$ & $\begin{array}{c}1 \\
29.5\end{array}$ & \\
\hline
\end{tabular}

Figure 4 Subgroup analysis of the risk of patients EAC and/or HGD in patients with BE. EAC, esophageal adenocarcinoma; HGD, High grade dysplasia; BE, Barrett's esophagus.

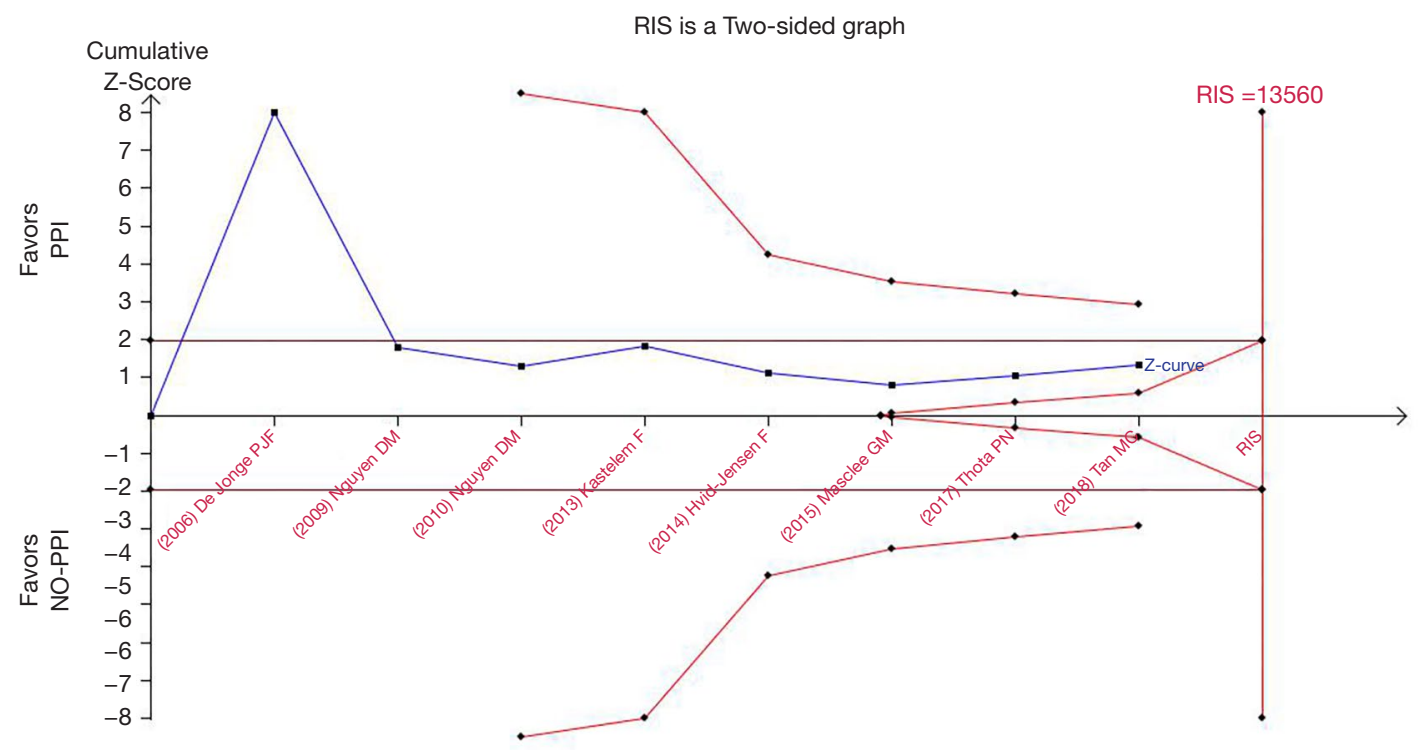

Figure 5 Trial Sequential Analysis of PPIs use and risk of EAC and/or HGD in patients with BE. EAC, esophageal adenocarcinoma; HGD, high grade dysplasia; BE, Barrett's esophagus; PPIs, Proton pump inhibitors; RIS, required information size. 
boundary for benefit was not crossed. The TSA of all trials showed that the amount of information accumulated was far from the amount of information needed and that more than 13,560 patients might be needed to draw firm conclusions. The overall pooled results showed no statistical difference (random-effects model: OR, 0.61; 95\% CI, 0.29-1.26).

\section{Discussion}

In a meta-analysis of 7,053 patients, PPIs' use to reduce the risk of HGD or EAC in BE was not confirmed, with significant heterogeneity being present. Our results are consistent with previous systematic assessments (18). Our analysis included additional procedures to provide a more reliable and comprehensive estimate of EC risk. With the increase in the number of participants, TSA was conducted to evaluate the statistical results' statistical value and guide further research.

$\mathrm{BE}$ is one of the complications of gastroesophageal reflux disease (GERD). Therefore, a reduction in gastric acid is generally the main strategy for treating patients with BE. Acid exposure has also been shown to upregulate the expression of cyclooxygenase-2 (COX-2) in $\mathrm{BE}$ (acid). COX-2 expression is increased during the early development of many tumors, including EAC, and is closely related to BE's development into EAC $(19,20)$. One meta-analysis showed that the use of COX inhibitors was negatively correlated with the risk of tumor progression in patients with $\mathrm{BE}$ (21), but these inhibitors' protective effect remains controversial. Other analyses have found contradictory results regarding the use of $\mathrm{H} 2$ receptor antagonists (H2RA) and the development of HGD or EAC, with some even reporting an increase in the risk of HGD or EAC $(4,22)$. Bile exposure has also been shown to upregulate the expression of COX-2 in BE (23). Interestingly, PPIs' main preventive mechanism is to promote the healing of esophageal mucosa by reducing esophageal acid and bile exposure.

Our meta-analysis did not find definite evidence supporting PPI intervention for improving the incidence of EAC in patients with BE. The results of our cohort study were different from those of our case-control study. One potential explanation for this result is the immortal time bias (24), which can produce illusory evidence for a therapeutic effect in some cohort studies.

PPIs have been shown to improve GERD in patients (25); however, whether or not the treatment of BE with PPIs reduces cancer risk remains unclear. Several randomized controlled trials have found that endoscopic therapy can effectively eliminate dysplasia and metaplastic epithelium, greatly reducing cancer incidence. Many treatments for BE, such as radiofrequency ablation, argon plasma coagulation, photodynamic therapy, endoscopic mucosal resection, and others have been applied. The presence of nodules, ulcers, or strictures in $\mathrm{BE}$ is thought to be associated with an increased risk of EAC (26). Once it occurs, patients' adverse effects may be minimized by removing the lesion rather than administering medication.

A few limitations to our study should be addressed. First, PPI dose effects, the timing of PPI use, and the occurrence of adverse events were not examined. Second, due to the limited number of studies analyzed, publication bias assessment was not conducted (27). However, we did conduct TSA to intuitively demonstrate that the treatment of $\mathrm{BE}$ by PPIs is still controversial and requires further exploration.

\section{Conclusions}

A meta-analysis and TSA of existing studies found no definitive evidence for PPIs' protective effect on the progression of $\mathrm{BE}$ patients to EAC or HGD. Indeed, the TSA suggests that more patients need to be included in the study before a clear conclusion can be reached.

\section{Acknowledgments}

Funding: This work was supported by the Zhejiang medicine key scientific and technology project (grant number: 2018258924) and the Zhejiang medicine scientific and technology project (grant number: No.2019RC094).

\section{Footnote}

Reporting Checklist: The authors have completed the PRISMA reporting checklist. Available at http://dx.doi. org/10.21037/tcr-20-3362

Conflicts of Interest: All authors have completed the ICMJE uniform disclosure form (available at http://dx.doi. org/10.21037/tcr-20-3362). The authors have no conflicts of interest to declare.

Etbical Statement: The authors are accountable for all aspects of the work in ensuring that questions related to the accuracy or integrity of any part of the work are 
appropriately investigated and resolved.

Open Access Statement: This is an Open Access article distributed in accordance with the Creative Commons Attribution-NonCommercial-NoDerivs 4.0 International License (CC BY-NC-ND 4.0), which permits the noncommercial replication and distribution of the article with the strict proviso that no changes or edits are made and the original work is properly cited (including links to both the formal publication through the relevant DOI and the license). See: https://creativecommons.org/licenses/by-nc-nd/4.0/.

\section{References}

1. Pohl H, Sirovich B, Welch HG. Esophageal adenocarcinoma incidence: are we reaching the peak? Cancer Epidemiol Biomarkers Prev 2010;19:1468-70.

2. Nguyen DM, Richardson P, El-Serag HB. Medications (NSAIDs, statins, proton pump inhibitors) and the risk of esophageal adenocarcinoma in patients with Barrett's esophagus. Gastroenterology 2010;138:2260-6.

3. Singh S, Garg SK, Singh PP, et al. Acid-suppressive medications and risk of oesophageal adenocarcinoma in patients with Barrett's oesophagus: a systematic review and meta-analysis. Gut 2014;63:1229-37.

4. García Rodríguez LA, Lagergren J, Lindblad M. Gastric acid suppression and risk of oesophageal and gastric adenocarcinoma: a nested case control study in the UK. Gut 2006;55:1538-44.

5. Moher D, Liberati A, Tetzlaff J, et al. Preferred reporting items for systematic reviews and meta-analyses: the PRISMA statement. PLoS Med 2009;6:e1000097.

6. Stang A. Critical evaluation of the Newcastle-Ottawa scale for the assessment of the quality of nonrandomized studies in meta-analyses. Eur J Epidemiol 2010;25:603-5.

7. Singh S, Singh AG, Singh PP, et al. Statins are associated with reduced risk of esophageal cancer, particularly in patients with Barrett's esophagus: a systematic review and meta-analysis. Clin Gastroenterol Hepatol 2013;11:620-9.

8. Guyatt GH, Oxman AD, Kunz R, et al. GRADE guidelines: 7. Rating the quality of evidence--inconsistency. J Clin Epidemiol 2011;64:1294-302.

9. DerSimonian R, Laird N. Meta-analysis in clinical trials. Control Clin Trials 1986;7:177-88.

10. Guyatt GH, Oxman AD, Kunz R, et al. What is "quality of evidence" and why is it important to clinicians? BMJ 2008;336:995-8.

11. de Jonge PJ, Steyerberg EW, Kuipers EJ, et al. Risk factors for the development of esophageal adenocarcinoma in Barrett's esophagus. Am J Gastroenterol 2006;101:1421-9.

12. Nguyen DM, El-Serag HB, Henderson L, et al. Medication usage and the risk of neoplasia in patients with Barrett's esophagus. Clin Gastroenterol Hepatol 2009;7:1299-304.

13. Kastelein F, Spaander MC, Steyerberg EW, et al. Proton pump inhibitors reduce the risk of neoplastic progression in patients with Barrett's esophagus. Clin Gastroenterol Hepatol 2013;11:382-8.

14. Hvid-Jensen F, Pedersen L, Funch-Jensen P, et al. Proton pump inhibitor use may not prevent high-grade dysplasia and oesophageal adenocarcinoma in Barrett's oesophagus: a nationwide study of 9883 patients. Aliment Pharmacol Ther 2014;39:984-91.

15. Masclee GM, Coloma PM, Spaander MC, et al. NSAIDs, statins, low-dose aspirin and PPIs, and the risk of oesophageal adenocarcinoma among patients with Barrett's oesophagus: a population-based case-control study. BMJ Open 2015;5:e006640.

16. Thota PN, Hajifathalian K, Benjamin T, et al. Lack of incremental effect of histamine receptor antagonists over proton pump inhibitors on the risk of neoplastic progression in patients with Barrett's esophagus: a cohort study. J Dig Dis 2017;18:143-50.

17. Tan MC, El-Serag HB, Yu X, et al. Acid suppression medications reduce risk of oesophageal adenocarcinoma in Barrett's oesophagus: a nested case-control study in US male veterans. Aliment Pharmacol Ther 2018;48:469-77.

18. Hu Q, Sun TT, Hong J, et al. Proton Pump Inhibitors Do Not Reduce the Risk of Esophageal Adenocarcinoma in Patients with Barrett's Esophagus: A Systematic Review and Meta-Analysis. PloS One 2017;12:e169691.

19. Spechler SJ, Sharma P, Souza RF, et al. American Gastroenterological Association technical review on the management of Barrett's esophagus. Gastroenterology 2011;140:e18-52; quiz e13.

20. Morris CD, Armstrong GR, Bigley G, et al. Cyclooxygenase-2 expression in the Barrett's metaplasiadysplasia-adenocarcinoma sequence. Am J Gastroenterol 2001;96:990-6.

21. Zhang S, Zhang XQ, Ding XW, et al. Cyclooxygenase inhibitors use is associated with reduced risk of esophageal adenocarcinoma in patients with Barrett's esophagus: a meta-analysis. Br J Cancer 2014;110:2378-88.

22. Chow WH, Finkle WD, McLaughlin JK, et al. The relation of gastroesophageal reflux disease and its treatment to adenocarcinomas of the esophagus and gastric 
cardia. JAMA 1995;274:474-7.

23. Shirvani VN, Ouatu-Lascar R, Kaur BS, et al. Cyclooxygenase 2 expression in Barrett's esophagus and adenocarcinoma: Ex vivo induction by bile salts and acid exposure. Gastroenterology 2000;118:487-96.

24. Suissa S. Immortal time bias in observational studies of drug effects. Pharmacoepidemiol Drug Saf 2007;16:241-9.

25. Zhang M, Pandolfino JE, Zhou X, et al. Assessing different diagnostic tests for gastroesophageal reflux disease: a systematic review and network meta-analysis. Therap Adv Gastroenterol 2019;12:1756284819890537.

26. Bujanda DE, Hachem C. Barrett's Esophagus. Mo Med 2018;115:211-3.

27. Sterne JA, Egger M, Smith GD. Systematic reviews in health care: Investigating and dealing with publication and other biases in meta-analysis. BMJ 2001;323:101-5.
Cite this article as: Li L, Cao Z, Zhang C, Pan W. Risk of esophageal adenocarcinoma in patients with Barrett's esophagus using proton pump inhibitors: a systematic review with metaanalysis and sequential trial analysis. Transl Cancer Res 2021;10(4):1620-1627. doi: 10.21037/tcr-20-3362 


\section{Appendix 1}

(((((()((((Barrett Esophagus) OR Barrett Epithelium) OR Barrett Metaplasia) OR Barrett Metaplasias) OR Barrett’s Syndrome) OR Barretts Syndrome) OR Barrett Syndrome) OR Barrett's Esophagus) OR Barretts Esophagus) OR high-grade dysplasia) OR oesophageal adenocarcinoma)) AND $(((((()(((($ proton pump inhibitor) OR pantoprazole) OR proton pumps) OR PPI) OR anti-ulcer agent) OR antacid) OR esomeprazole) OR omeprazole) OR ilaprazole) OR dexlansoprazole) OR rabeprazole) OR lansoprazole) OR acid suppress*) 